$\S=-1$

\title{
Synthesis of Linear Array Antenna with Wide Range of beamwidths using DE Algorithm
}

\author{
Challaram Grandhi ${ }^{1 *}$, N.Venkateswara rao ${ }^{2}$ \\ ${ }^{1}$ M.Tech, Department of ECE, SRKR Engineering College, Bhimavaram. \\ ${ }^{2}$ Professor, Department of ECE, SRKR Engineering College, Bhimavaram. \\ *Corresponding author E-mail:challaram.grandhi@gmail.com
}

\begin{abstract}
In many communication systems like Radar and point to point micro links there is a need of both narrow and wide beams. In target applications Antennas with narrow beam width is required on the other hand, for spy application antennas with wider beam width are required. Generally, for linear array antennas the beams width will be narrowed by increase in the number of elements in the array. To obtain narrow beams without a significant increase in the number of radiation elements in the array, the optimization algorithms are used. By the use of optimization algorithms the wider beams can also be employed in reverse procedure of choosing the highest output for a given set of random inputs. These wide ranges of beam width are obtained without any increase in the side lobe levels that is, by maintaining the side lobe level below -20db. Random stochastic methods like Particle swarm optimization (PSO), Genetic algorithm (GA), Biogeography-based optimization (BBO) and Differential Evolution Algorithm (DE) are used and the results are compared.
\end{abstract}

Keywords: Biogeography-based optimization (BBO); Differential Evolution Algorithm (DE); First Null Beamwidth (FNBW); Genetic Algorithm (GA); Particle Swarm Algorithm(PSO); Sidelobe level (SLL).

\section{Introduction}

In many communication systems there is a need for high directive beam with very low side lobe levels (SLL), in order to achieve this several excitation elements are placed in form of an array. Linear array antenna is a set of elements placed at uniform spacing between excitation elements. The side lobe radiates the unwanted radiations, which may lead to interference of other equipments. On the other hand the receiving antenna side lobes may receive the interfering signals and increase the noise level in the received signals. An efficient receiver and transmitter require a decrease in the side lobe level [4]. For the reduction of side lobe levels optimization of SLL is performed with random stochastic methods like genetic algorithm, Particle swarm algorithms, etc.

All these wide range of beam widths are obtained without significant increase in the side lobe level. The side lobe level is maintained below -20db. The optimization algorithms [1] like Particle swarm optimization (PSO) , Genetic algorithm (GA), Biogeography-based optimization (BBO) and Differential Evolution Algorithm (DE) are used to obtain the narrow beams and wide beams without increase in side lobe level and number of radiation elements in array. The results of all these optimization techniques are compared.

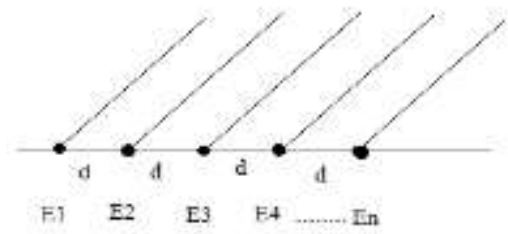

Fig. 1: Linear Array Antenna
Fig.1 indicates a linear array antenna with $\mathrm{N}$ number of elements placed at a distance $\mathrm{d}$ from each other. The excitation of each element is given by $E_{m}$ where $m=1,2, \ldots . n$. the excitation of $m$ th element is given by $\mathrm{E}_{\mathrm{m}}$. For isotropic elements the radiation pattern can be given by their array factor.

Excitation of the elements in uniform linear array can be given by

$$
E=1+e^{j \varphi}+e^{j 2 \varphi}+e^{j 3 \varphi}+\ldots \ldots . .+e^{j(n-1)}
$$

Where

$\varphi=2 \pi\left(\frac{d}{\lambda}\right) \cos \phi+\alpha$

Array factor of linear array antenna is given by

$$
A F=E_{0} e^{j \beta z_{0} \cos \theta}+E_{1} e^{j \beta z_{1} \cos \theta}+\ldots \ldots . .+E_{N-1} e^{j \beta z_{N-1} \cos \theta}
$$

\section{Optimization Algorithms}

An easy The optimization algorithms like Particle swarm optimization (PSO), Genetic algorithm (GA), Biogeography-based optimization (BBO) and Differential Evolution Algorithm (DE) are used to obtain the narrow and wide beams without increase in side lobe level and number of radiation elements in array. The results of all these optimization techniques are compared.

\subsection{Genetic Algorithm (GA)}

Genetic algorithm is the most commonly used optimization technique. As the name it indicates that this algorithm deals with the 
genes. To obtain best outcomes these genes undergoes several manipulations like crossover and mutation [2]. The parent genes are first selected form population and then these parent genes undergoes cross over which indicates the exchange of genes among parents to produce high quality genes which are termed as child genes.

General steps involved in genetic algorithm are

- $\quad$ select parent chromosomes

- crossover of child chromosomes

- mutation of child chromosomes

- $\quad$ check termination condition

These steps indicate a clear order of execution of genetic algorithm. Here at each and every stage the termination criteria must be checked to proceed. The fitness function used for this algorithm should be choosing in order to obtain best beam width values [3]

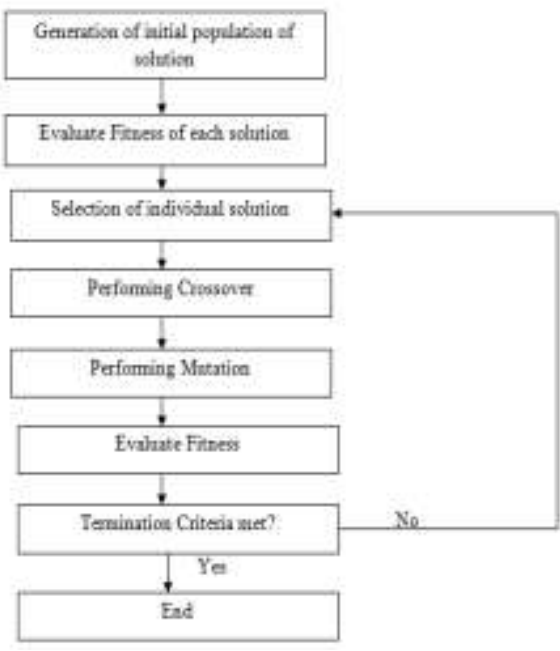

Fig. 2: Flow chart for Genetic Algorithm

\subsection{Particle Swarm Optimization (PSO)}

PSO optimization is one of the well known computational methods in computer science which optimizes a problem iteratively to obtain the best results. As the name of the algorithm indicates that the particles will swarm around the space. In this algorithm it deals with the population where each member will act as a particle and move in the space according to the formulas. As all the particles are in motion the parameters such as position and velocity are always modified by the mathematical equations stated in the algorithm. By modifying these parameters the improved position and velocities will guide the particles and this process is continued till the satisfactory results are obtained.

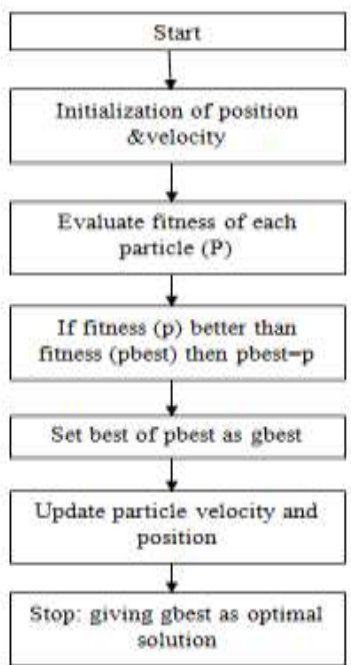

Fig. 3: Flow chart for PSO Algorithm

\subsection{Biogeography-Based Optimization (BBO)}

Like PSO, BBO is also an evolutionary algorithm which is computed iteratively. It is generally used to optimize multidimensional valued function. This algorithm examines the migration of species between islands and the presence of species. Islands with high habitat suitability index (HSI) are friendly to live. Islands with a high HSI not only have a high emigration rate, but they also have a low immigration rate because they already support many species. Species that migrate to such islands will tend to die in spite of the island's high HSI, because there is too much competition for resources from other species. Islands with a low HSI have a high immigration rate because of their low populations. Steps involved in BBO technique are selection of population, examine immigration and emigration rates, migration and mutation operation, replace worst habitants, update the best values, and check termination criteria. These steps are repeated for certain number of iterations or until the termination criteria is obtained.

\section{Proposed Methodology}

In this paper the proposed methodology deals with a quick and efficient optimization technique named Differential Evolution Algorithm (DE) [7]. This proposed methodology is similar to the above optimization apart of using mutation as primary search algorithm. The main advantage of this algorithm is that it repeatedly uses the mutation for the given random inputs to obtain the best output for the given inputs [6].

- Initially the inputs are obtained randomly.

- Obtain random permutations of given inputs to generate new set of inputs.

- Mutate the new set of values. That is round the values above and below the predefined values.

- After mutation the results are sent for crossover which is similar to cross over in genetic algorithm.

- The new set of inputs obtained after crossover are used as input to algorithm.

- Definition of Fitness functions or cost functions

- $\quad \operatorname{Cost}=\left(\mathrm{FNBW}_{\text {desired }}-\mathrm{FNBW}_{\text {obtained }}\right)$

- Obtain the set of outputs for the given fitness function.

- Compare the outputs of fitness function and store the best values.

- Steps are repeated iteratively.

- Update the best values at the end of each iteration.

- Best value is taken as output.

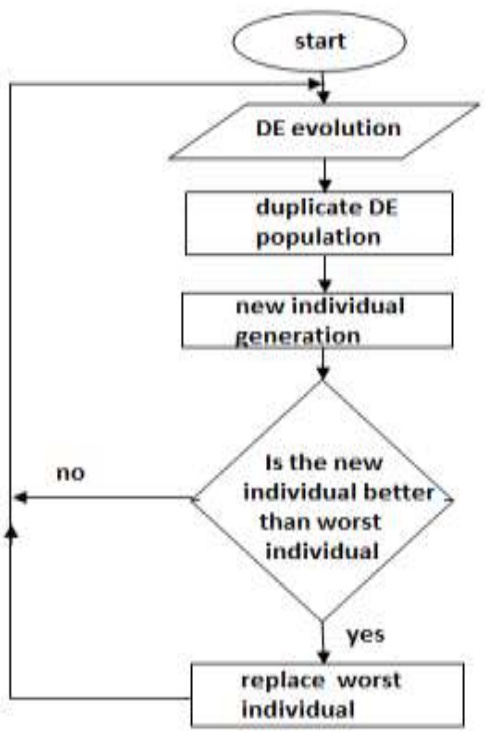

Fig. 4: Flow chart for Proposed Algorithm (DE) 
Fig.2 indicates a clear order of execution of DE algorithm. Here at each and every stage the termination criteria must be checked to proceed. The fitness function used for this algorithm should be choosing in order to obtain best beam width values [7].

\section{Results}

Implementation of these algorithms is carried on MATLABR2013a software. The results are obtained for the optimization of First null beam width (FNBW) and side lobe levels (SLL). A wide range of beam widths are obtained for a linear array antenna with uniform distribution [5]. All the antennas are placed uniformly in linear fashion for these results the spacing between the antennas is remained constant and the value is half the lambda i.e. $\mathrm{d}=\left(0.5^{*}\right.$ lambda $)$.

For all these results the side lobe level is set to be below $-20 \mathrm{db}$ so that the synthesis of the linear array antenna is possible practically without any interference of noisy signals. Results are obtained so that antenna can be synthesized with a range of beam width of order 4 to 125 degrees with number of radiation elements within range of 10 to 30 .

\subsection{Results for Uniform Linear Array Antenna}

For uniform linear array the amplitude and the spacing between the radiation elements is remained constant. The below results are obtained by placing all the elements with unity amplitude and half the lambda spacing. The results before optimization are tabulated as below.

Table 1: Results of FNBW for uniform linear array antenna.

\begin{tabular}{|c|c|c|c|}
\hline $\begin{array}{c}\text { No.of elements } \\
(\mathrm{N})\end{array}$ & $\begin{array}{c}\text { Spacing (d) } \\
\text { in lambda }\end{array}$ & SLL in db & $\begin{array}{c}\text { FNBW in de- } \\
\text { grees }\end{array}$ \\
\hline 30 & 0.5 & -13.26 & 9.2 \\
\hline 20 & 0.5 & -13.19 & 12.4 \\
\hline 16 & 0.5 & -13.14 & 16.2 \\
\hline 10 & 05 & -12.96 & 22.9 \\
\hline
\end{tabular}

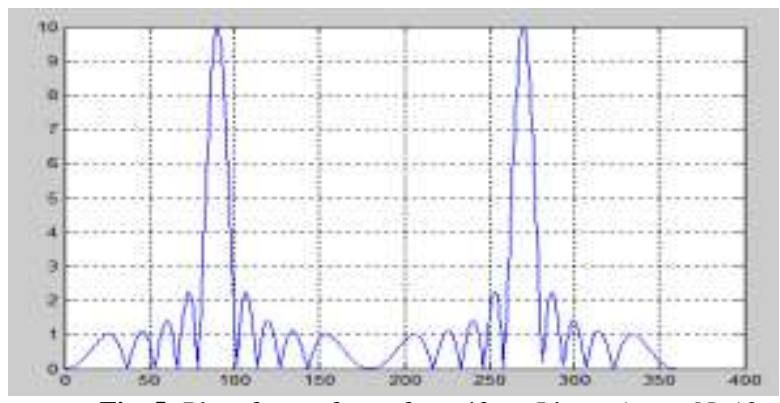

Fig. 5: Plot of array factor for uniform Linear Array, $N=10$

Fig. 5 gives the plot of array factor for $\mathrm{n}=10$ with uniform distribution and uniform excitation.

\subsection{Results of FNBW Using Optimization Algorithms}

The results obtained from optimization algorithms like GA, PSO, BBO and Proposed methodology are tabulated and compared, all these results are obtained for a linear array with uniform spacing between elements and non uniform amplitudes i.e. these amplitudes are generated randomly.

To obtain a range of beam widths of range 5-20 degrees with minimum number of elements there is a need for positive optimization which is the traditional method of obtaining the best minimal value. By use of optimization algorithms a decrease in the beamwidth can be obtained.
Table 2: Results of FNBW using positive optimization algorithms.

\begin{tabular}{|c|c|c|c|c|c|}
\hline $\begin{array}{c}\text { No.of } \\
\text { elements } \\
(\mathrm{N})\end{array}$ & $\begin{array}{c}\text { SLL } \\
\text { in db }\end{array}$ & $\begin{array}{c}\text { FNBW } \\
\text { in de- } \\
\text { grees } \\
\text { Using } \\
\text { GA }\end{array}$ & $\begin{array}{c}\text { FNBW } \\
\text { in de- } \\
\text { grees } \\
\text { Using } \\
\text { PSO }\end{array}$ & $\begin{array}{c}\text { FNBW } \\
\text { in de- } \\
\text { grees } \\
\text { Using } \\
\text { BBO }\end{array}$ & $\begin{array}{c}\text { FNBW in } \\
\text { degrees } \\
\text { Using pro- } \\
\text { posed meth- } \\
\text { odology }\end{array}$ \\
\hline 30 & -24.08 & 6.8 & 6.4 & 5.2 & 4.8 \\
\hline 20 & -23.84 & 9.4 & 8.8 & 7.4 & 6.8 \\
\hline 16 & -22.38 & 14.2 & 12.0 & 8.8 & 8.0 \\
\hline 10 & -21.97 & 18.3 & 16.8 & 14.2 & 12.6 \\
\hline
\end{tabular}

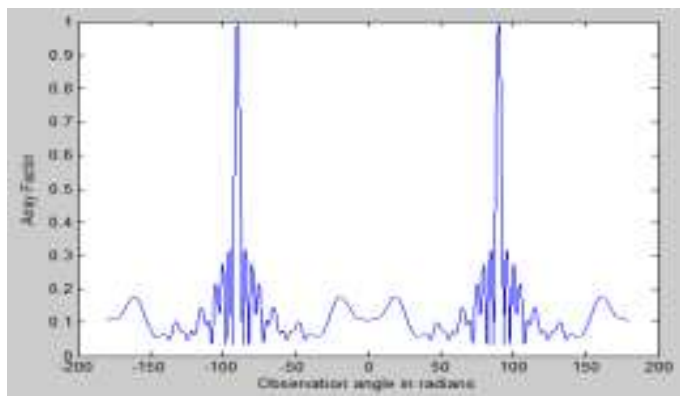

Fig. 6: Plot of array factor for non uniform Linear Array, $N=30$ (DE)

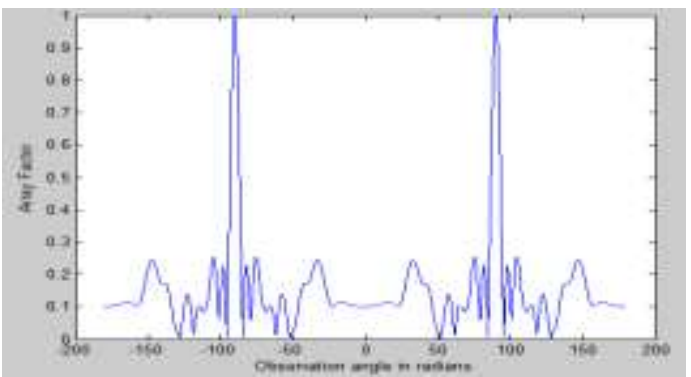

Fig. 7: Plot of array factor for non uniform Linear Array, N=30 (PSO)

Fig. 6 and Fig. 7 gives the plot of array factor for $n=30$ with uniform distribution and non uniform excitation using DE and PSO optimization algorithms respectively.

To obtain a range of beam widths of range 20-125 degrees with minimum number of elements there is a need for negative optimization which is different to traditional algorithm by choosing best maximal values. By use of the negative optimization algorithms an increase in the beamwidth can be obtained. All the values are tabulated as below.

Table 3: Results of FNBW using negative optimization algorithms.

\begin{tabular}{|c|c|c|c|c|c|}
\hline $\begin{array}{c}\text { No.of } \\
\text { elements } \\
(\mathrm{N})\end{array}$ & $\begin{array}{c}\text { SLL } \\
\text { in db }\end{array}$ & $\begin{array}{c}\text { FNBW } \\
\text { in de- } \\
\text { grees } \\
\text { Using } \\
\text { GA }\end{array}$ & $\begin{array}{c}\text { FNBW } \\
\text { in de- } \\
\text { grees } \\
\text { Using } \\
\text { PSO }\end{array}$ & $\begin{array}{c}\text { FNBW } \\
\text { in de- } \\
\text { grees } \\
\text { Using } \\
\text { BBO }\end{array}$ & $\begin{array}{c}\text { FNBW in } \\
\text { degrees } \\
\text { Using pro- } \\
\text { posed meth- } \\
\text { odology }\end{array}$ \\
\hline 30 & -28.43 & 13.6 & 15.2 & 32.6 & 39.8 \\
\hline 20 & -27.32 & 22 & 24.2 & 60.6 & 72.4 \\
\hline 16 & -25.67 & 29.5 & 32.4 & 76.2 & 83.6 \\
\hline 10 & -24.89 & 48.5 & 61.6 & 109.3 & 125.2 \\
\hline
\end{tabular}

From Table. 3 beamwidth is increased up to 125 degrees and from Table. 2 beamwidth is decreased to 4.8 degrees thus a wide range of beamwidths are obtained using proposed algorithm.

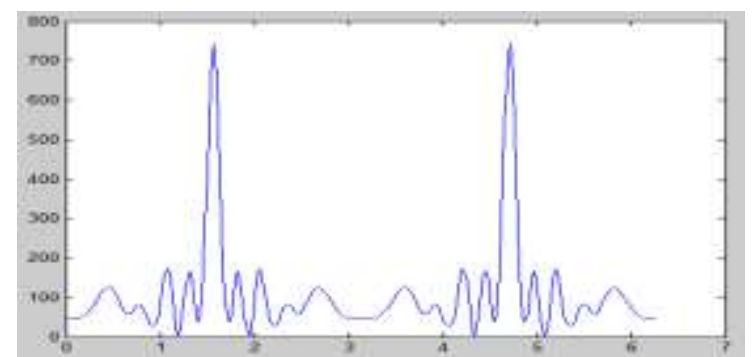

Fig. 8: Plot of array factor for non uniform Linear Array, $N=10$ (DE) 


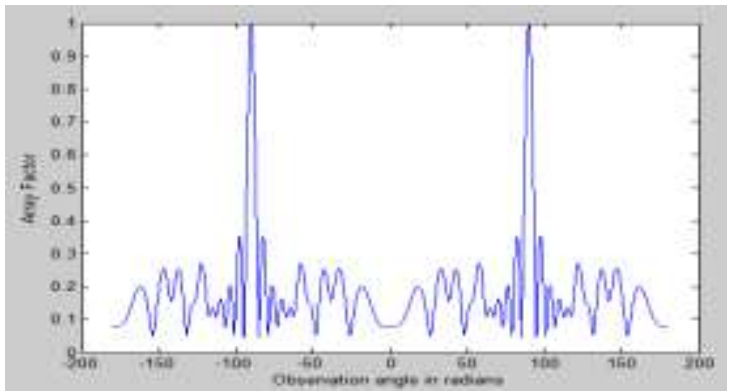

Fig. 9: Plot of array factor for non uniform Linear Array, N=10 (BBO)

Fig. 8 and Fig. 9 gives the plot of array factor for $\mathrm{N}=10$ with uniform distribution and non uniform excitation using DE and $\mathrm{BBO}$ optimization algorithms respectively.

\section{Conclusion}

In this paper optimization of SLL and FNBW of linear antenna array is done with uniform spacing and non-uniform excitation. By using proposed algorithm for linear array antenna FNBW is reduced up to 4.8 degrees and increased up to 125.2 degrees. A narrow beam and wide beam with low sidelobe level is obtained by using DE algorithm and values are compared with GA, PSO and $\mathrm{BBO}$ algorithms.

An antenna with wide range of beamwidth from 5 degrees to 125 degrees can be synthesized by using proposed methodology with less number of elements and also with low sidelobe level. From the results it is clear that proposed method is far superior to GA, $\mathrm{PSO}$ and $\mathrm{BBO}$ algorithm.

\section{References}

[1] Iqroop Kaur, Sanjeev Kumar "linear antenna array optimization techniques", International Journal of Engineering Trends and Technology- May to June Issue 2011, ISSN: 2231-5381 - 1 - IJETT.

[2] Biul Goswami, Durbadal Mandal, "A genetic algorithm for level control of nulls and side lobes in linear antenna arrays", Journal of king sand university- computer and information sciences.

[3] Padmaja, N.Bala Subramanyam, N.Deepika Rani, G.Tirumala Rao, Linear Array Geometry Synthesis Using Genetic Algorithm for Optimum Side Lobe Level and Null, ISSN: 2277 - 9043 International Journal of Advanced Research in Computer Science and Electronics Engineering Volume 1, Issue 3, May 2012.

[4] C. A. Balanis, Antenna Theory Analysis and Design, 2nd Edition, John Willy \& sons Inc, New York, 1997

[5] Shraddha shrivastava and Kanchan cecil, "Optimatization of radiation pattern of linear antenna array using genetic algorithm" Recent Trends in Control, Communication and Computer Technology 25th Nov.2012, Nashik, ISBN: 978-93-82208-40-2.

[6] Stephen J.Blank, “Antenna Array Synthesis Using Derivative, NonDerivative and Random Search Optimization", IEEE Sarnoff Symposium, DOI 10.1109/SARNOF. 2008.4520115, pp 1-4, May 2008.

[7] T.prakash, V.P. Singh, D.P.S. Chauhan "Computational Intelligence \& Communication Technology (CICT), 2016 Second International Conference", IEEE DOI: 10.1109/CICT.2016.60. Feb, 2016. 